\title{
Gamificação e avaliação do engajamento dos estudantes em uma disciplina técnica de curso de graduação
}

\author{
Sérgio A. A. Freitas ${ }^{1}$, Thiago Lima ${ }^{1}$, Edna Dias Canedo ${ }^{1}$, Ricardo Lopes Costa ${ }^{2}$ \\ ${ }^{1}$ Faculdade UnB Gama - Universidade de Brasília (UnB) \\ Area Especial Industria Proj. A, Gama - Brasília - DF - Brasil \\ ${ }^{2}$ Funifier - Rua das Carnaúbas, Lote 2, $5^{\circ}$ Andar, Águas Claras, \\ CEP 71904-540, Brasília - DF - Brasil \\ \{SergioFreitas,EdnaCanedo\}@unb.br, \{thiagolima.unb\}@gmail.com, \\ Ricardo@funifier.com
}

\begin{abstract}
The gamification of activities other than games has become one of the main objectives of a new area of research. In the education area the proposal could not be different: the new generations entering the higher education has a lot of experience in the virtual information environment and games. So, nothing more natural than checking the adherence of gamification to teaching this new student profile. In this scenario, this article presents a case study of a gamification for a discipline of an undergraduate course. The gamification space was built based on a framework that stands on basic human motivations. Finally, it made a statistical evaluation of the engagement and motivation of students with the introduction of gamification in the discipline.
\end{abstract}

Resumo. A gamificação de atividades que não sejam jogos tornou-se um dos principais objetivos desta nova área de pesquisa. Na educação, a proposta não poderia ser diferente: com as novas gerações ingressando no ensino superior e já com uma vivência no ambiente virtual de informação e jogos, nada mais natural do que verificar-se a aderência da gamificação ao ensino deste novo perfil de estudante. Neste cenário, este artigo apresenta um estudo de caso de um espaço de gamificação para uma disciplina de um curso de graduação. $O$ espaço foi construído tomando por base um framework de gamificação por motivações básicas. Por fim, foi feito uma avaliação estatística do engajamento e motivação dos alunos com a introdução da gamificação na disciplina.

\section{Introdução}

Parece ser senso comum que bons professores motivam seus estudantes. Entretanto, será que ensinar melhor através de aulas expositivas é a única maneira pela qual um aprendiz pode se sentir motivado a aprender? Uma resposta alternativa é a aprendizagem ativa (Briggs, 2005), onde é proposto um modelo de ensino focado na responsabilidade da aprendizagem por parte dos aprendizes. Na aprendizagem ativa, os estudantes estão inseridos num ambiente de aprendizagem no qual os três domínios de Bloom (Bloom, 1956) são estimulados, a saber: conhecimento, aptidões e atitudes. 
V Congresso Brasileiro de Informática na Educação (CBIE 2016)

Anais do XXVII Simpósio Brasileiro de Informática na Educação (SBIE 2016)

$\mathrm{Na}$ atualidade, as possibilidades de aprendizagem têm se tornado cada vez mais atrativa. As novas gerações têm feito um uso intensivo das tecnologias digitais modernas, tanto em termos de dispositivos: computadores, celulares e vídeo games, quanto de aplicativos: Facebook, Whatsapp, compartilhamento de conteúdos, entre outros (McGonigal, 2011). Esta geração é nativamente digital (Prensky, 2007) e tem uma "aptidão nata" para aprender interagindo (McGonigal, 2011). Estes novos "usuários" preferem, de forma autônoma, descobrir os conteúdos e aprendem mesmo estando imersos "no mar de informações" da internet. Jogar é uma atividade corriqueira para estas gerações.

Neste cenário, a gamificação é uma nova área de pesquisa que preconiza o uso de elementos de desenho e princípio dos jogos para aumentar o engajamento, a motivação e o prazer dos usuários na execução de tarefas diárias (Kumar \& Herger, 2013; Chou, 2015; Vianna, Vianna, Medina, \& Tanaka, 2013). Algumas práticas de gamificação estão sendo utilizadas em contextos fora do ambiente dos jogos: $\mathrm{Na}$ produtividade organizacional (McGonigal, 2011), na aprendizagem (Kapp, Blair, \& Mesch, 2014; Freitas S. A., Silva, Lucena, RIBEIRO, LIMA, \& SILVA, 2016) e no recrutamento, avaliação e treinamento de empregados (Vianna, Vianna, Medina, \& Tanaka, 2013).

No Brasil, a gamificação está iniciando seus passos e já conta com alguns trabalhos interessantes: Figueiredo et al (Figueiredo, Paz, \& Junqueira, 2015) e Borges et al (Borges, Reis, Durelli, Bittencourt, Jaques, \& Isotani, 2013) apresentam levantamentos das pesquisas em gamificação e educação, Ellwager et al (Ellwanger, Santos, \& Moreira, 2014) demonstram as relações entre a gamificação, os padrões de interface e a mobilidade no desenvolvimento de aplicações educacionais e Seixas et al (Seixas, Gomes, Filho, \& Rodrigues, 2014) apresentam um estudo de caso em uma escola do ensino fundamental.

O uso de Gamificação na educação das novas gerações figura uma opção natural, desde o ensino fundamental até o ensino superior. A gamificação é, em si, uma metodologia de aprendizagem ativa em que os alunos podem se tornar aprendizes engajados e motivados nas atividades de aprendizagem.

$\mathrm{O}$ desafio é criar um ambiente gamificado no espaço da sala de aula e fora dela. $\mathrm{O}$ espaço de gamificação, aqui proposto, é um jogo intitulado "FAC - o jogo: Batalha do conhecimento". Ele foi projetado, implementado e testado na disciplina de Fundamentos de Arquitetura de Computadores (FAC) da Faculdade UnB Gama (FGA) da Universidade de Brasília (UnB). FAC é uma disciplina do quarto semestre oferecida simultaneamente aos cursos de graduação em Engenharia de Software e em Engenharia Eletrônica.

O objetivo deste artigo é apresentar este espaço criado na disciplina, bem como a avaliação do engajamento e motivação dos alunos a partir da introdução da gamificação. FAC - o jogo foi pautado na proposta apresentada por McGonigal (McGonigal, 2011) e no framework Octalysis de Yu Kai-Chou (Chou, 2015).

Esta pesquisa empírica e exploratória está assim estruturada: na seção 2 é apresentado o referencial teórico utilizado, na seção 3 é apresentada a descrição das principais características da disciplina de Fundamentos de Arquitetura de Computadores, na seção 4 é apresentado o detalhamento do jogo e sua execução, na seção 5 a avaliação do 
V Congresso Brasileiro de Informática na Educação (CBIE 2016)

Anais do XXVII Simpósio Brasileiro de Informática na Educação (SBIE 2016)

engajamento e da motivação dos alunos com a introdução da gamificação e, por fim, na seção 6 são apresentadas as conclusões e alguns trabalhos futuros.

\section{Referencial Teórico}

De acordo com McGonigal (McGonigal, 2011) existem quatro características que determinam um jogo: meta, regras, feedback e participação voluntária.

A meta indica o objetivo pelo qual um jogador deseja jogar o jogo. As regras indicam as normas, restrições e ações permitidas que norteiem o jogador no seu percurso dentro do jogo. O feedback são as respostas do jogo a cada ação executada, indicando o quanto o jogador está distante ou não da meta do jogo. Já a participação voluntária é a liberdade que o jogador tem de tentar ou não o jogo. Na sequência, caso esteja jogando, envolve também a liberdade de escolha dos seus próprios caminhos e estratégias dentro do jogo.

Partindo desta conceituação, foi criado um espaço gamificado onde o estudante vê a disciplina como se fosse um jogo: a meta é concluir a disciplina com aproveitamento, as regras são a ementa, o plano de ensino e a descrição do jogo, os feedbacks são as respostas às perguntas do jogo, a interação com outros estudantes e os "ganhos" resultantes das vitórias conseguidas durante o jogo. Por fim, a disciplina é optativa para ambos os cursos, assim a participação é feita de forma voluntária quando da matrícula da disciplina.

De posse do roteiro proposto por McGonigal (McGonigal, 2011), o próximo passo é mapear quais seriam as motivações e as técnicas de jogos a serem utilizadas na construção do jogo. O framework Octalysis proposto por Yu Kai-Chou (Chou, 2015) apresenta oito motivações básicas que levam os jogadores a jogarem e mapeia uma ampla gama de técnicas de jogos (game techniques) associada a cada das motivações básicas.

Segundo Chou (Chou, 2015) as motivações básicas dos jogadores, chamadas por ele de Core Drivers, são:

1. Significado Épico \& Chamado - quando o jogador acredita que está fazendo algo maior, por um bem maior ou que foi "escolhido" para fazer alguma coisa transcendental.

2. Desenvolvimento \& Realização - quando o jogador observa seus avanços, progressos, desenvolvimento de habilidades e, eventualmente, a superação de desafios.

3. Empoderamento \& Feedback - quando o jogador está envolvido num processo criativo onde repetidamente tem de descobrir "coisas"1 e tentar combinações diferentes.

4. Propriedade \& Posse - quando o jogador é motivado porque tem a sensação de posse ou propriedade de alguma "coisa".

5. Influência Social \& Pertencimento - quando o jogador é motivado por elementos sociais que influenciam as pessoas, incluindo: orientação, aceitação, respostas sociais, companheirismo, bem como a competição e a inveja.

\footnotetext{
${ }^{1}$ O termo "coisa" é intencionalmente vago. Pode ser um bem material, uma sensação, um objetivo abstrato, um desejo etc.
} 
V Congresso Brasileiro de Informática na Educação (CBIE 2016)

Anais do XXVII Simpósio Brasileiro de Informática na Educação (SBIE 2016)

6. Escassez \& Impaciência - quando o jogador é motivado pelo desejo de algo que não pode ter.

7. Imprevisibilidade \& Curiosidade - quando o jogador é motivado por querer descobrir o que vai acontecer a seguir. Se ele não sabe o que vai acontecer, o seu cérebro está envolvido e pensa sobre isso muitas vezes.

8. Perda \& Rejeição - quando o jogador é motivado pela prevenção de algo negativo que possa ocorrer.

Para cada uma destas motivações básicas, Chou (Chou, 2015) apresenta e categoriza um conjunto de técnicas de jogo (73 técnicas). A proposta é que a aplicação de uma dada técnica irá induzir e fortalecer uma dada motivação na gamificação. Alguns exemplos de técnicas são: Narrativa (\#10), Sorte de principiante (\#23), Elitismo (\#26) entre outras.

Como resultado, um estudante com um dado perfil: competitivo, colaborativo, ansioso, solidário entre outros, está em sintonia com um conjunto de motivações. Ao se utilizar técnicas que fortaleçam as motivações correspondentes, haverá um forte engajamento do estudante nas atividades do jogo, com a produção de uma sensação de atividades gratificantes e recompensadoras.

$\mathrm{Na}$ gamificação aqui apresentada, foram utilizadas as motivações básicas: Significado Épico e Chamado, Desenvolvimento \& Realização, Imprevisibilidade \& Curiosidade e Empoderamento \& Feedback.

\section{A disciplina FAC}

Fundamentos de Arquitetura de Computadores (FAC) é uma disciplina de 60 horas, optativa, ofertada no $4^{\circ}$ semestre dos cursos de graduação em Engenharia de Software e de Engenharia Eletrônica da FGA/UnB. Nos últimos 10 semestres houve uma média 33 estudantes por turma semestral. O professor foi o mesmo durante este período. Os livros textos utilizados são: Organização Estrutura de Computadores (Tanenbaum, 2013), Computer Organization and Design (Patterson \& Hennessy, 2013) e Computer Organization and Architecture (Stallings, 2012).

\subsection{Objetivo da disciplina}

O objetivo primário de FAC é cognitivo (Bloom, 1956): demonstrar ao estudante como um programa de computador é executado pelo hardware de um computador, em especial, apresentar como uma macro instrução pode ser executada por uma CPU. No final do curso, espera-se que o estudante entenda a organização interna da CPU, os seus componentes e como eles interagem para produzir a execução de um programa.

\subsection{Metodologia de avaliação}

A metodologia padrão de avaliação da disciplina tem sido contínua e, preferencialmente, revisada pelos pares. Cabe salientar que a disciplina já testou outras metodologias de ensino/aprendizagem: Aprender ensinando (Fiorella \& Mayer, 2014; Freitas, Silva, \& Marsicano, 2016) e Aprendizagem baseada em problemas (PBL) (Bell, 2010). Em todas, as metodologias o processo de avaliação foi sempre o mesmo. 
Com a introdução da gamificação é que o processo de avaliação tornou-se diário. Em cada aula o aluno tem um desempenho (em termos de ganhos no jogo) que reflete automaticamente a avaliação da sua aprendizagem naquela aula. É possível, inclusive, acompanhar o percurso de aprendizagem de cada estudante individualmente durante o semestre. As tradicionais provas (pontuais) não existem em FAC. Independente da metodologia, o resultado final é traduzido para os valores de aprovação e reprovação institucionais, onde $50 \%$ ou mais de aproveitamento indica aprovação na disciplina.

\section{O espaço gamificado de $\mathrm{FAC}$ - o jogo}

O espaço gamificado é constituído pelos seguintes elementos: o espaço físico (sala de aula), o espaço virtual (ambiente moodle), as fontes de informação (livros, vídeos e internet), os jogadores (estudantes, professor e monitores), a definição da nomenclatura e o jogo. A seguir alguns destes itens são detalhados.

\subsection{A sala de aula}

A sala de aula é um elemento importante na gamificação apresentada, dado que o jogo será jogado, em grande parte, no espaço da sala. Nele os estudantes devem ter facilidade de movimentação, boa acústica e iluminação, bem como disponível um quadro com material para escrita no mesmo. Não são necessários projetores de multimídia ou computadores. São altamente desaconselháveis o acesso à internet e a utilização de smartphones.

\subsection{O espaço virtual}

O planejamento, o controle e a comunicação da gamificação são executados no espaço virtual, no caso em questão, o moodle institucional. Neste espaço está o cronograma das aulas, a lista de tópicos de cada aula, a disponibilização de materiais (vídeos), a relação de atividades extraclasse, todo o material produzido no jogo (para dar transparência aos resultados) e, por fim, o ranking dos jogadores.

\subsection{Definição da nomenclatura}

Para que a gamificação seja efetiva, é necessário desvincular os jogadores do mundo real. Assim as nomenclaturas tradicionais devem ser substituídas. Termos como aula, professores, prova entre outros, devem ser banidos do vocabulário dos estudantes, dos professores e dos monitores. A nomenclatura utilizada passou a ser: Disciplina = jogo, aula $=$ batalha, estudante $=$ jogador, professor $=$ oráculo, monitor $=$ pitonisa, pontos $=$ patacas, avaliações dentro da aula $=$ duelos, avaliações após a aula $=$ desafios e, finalmente, provas/trabalhos $=$ missões .

\section{$4.4 O$ jogo}


V Congresso Brasileiro de Informática na Educação (CBIE 2016)

Anais do XXVII Simpósio Brasileiro de Informática na Educação (SBIE 2016)

O elemento central do jogo é o duelo de conhecimento entre os jogadores (individual ou em grupo). O conjunto de duelos constitui uma batalha. Cada batalha discorre sobre um ou mais tópicos da disciplina estão previamente disponibilizados no ambiente virtual. $\mathrm{O}$ conjunto de batalhas cobre todos os tópicos da disciplina. Para que os jogadores tenham êxito nos duelos é necessária uma preparação prévia com estudo nas fontes disponíveis.

Após cada batalha, fica disponível no ambiente virtual um conjunto de desafios que o jogador por realizar e assim ganhar mais patacas. A cada mês são disponibilizadas missões que devem ser realizadas pelos jogadores no decorrer de uma semana. Todas as atividades do jogo: duelos, desafios e missões que sejam realizadas com sucesso dão origem a patacas (moeda do jogo). O objetivo do jogo é acumular patacas. No final do jogo, cada jogador mapeia suas patacas em uma nota final.

Foram utilizadas as seguintes técnicas de Chou (Chou, 2015): Narrativa, Elitismo, Leaderboard, Choice perception, Meaningful choices, Exchangeable points, Mentorship, Group quest, Social Treasures, Social Proud, Torture break, Glowing choice, Easter eggs, FOMO punch, Rolling rewards e Countdown timer.

\section{O duelo}

Um duelo consiste em uma disputa de pergunta e resposta entre os jogadores. Um grupo (desafiante) de jogadores formula uma pergunta referente ao tema da batalha para o outro grupo (desafiado) responder. $\mathrm{O}$ oráculo faz o papel de árbitro e julga o quanto vale a pergunta. Os valores possíveis são de uma a três patacas, dependendo da complexidade da pergunta feita. O tempo destinado a cada duelo é de cinco minutos.

O grupo desafiado ganha as patacas (total ou parcialmente) ao responder satisfatoriamente a pergunta. $\mathrm{O}$ grupo desafiante ganha patacas caso a resposta não seja satisfatória. As patacas ganhas são divididas entre os membros do grupo. A cada batalha existe um limite de ganho de 6 patacas por jogador.

Os demais jogadores que não estão participando do duelo podem complementar tanto a pergunta, quanto a resposta. Caso uma pergunta não tenha sido respondida completamente, qualquer jogador fora do duelo pode complementar a resposta e ganhar a pontuação complementar. Caso o grupo desafiado não responda satisfatoriamente, os jogadores na plateia podem complementar a resposta e ganhar uma pataca. A final do duelo, o desafiante deve responder corretamente à pergunta realizada sob pena do duelo ser invalidado. A formação dos grupos é definida pelo oráculo no início de cada batalha, tanto em relação à quantidade de membros quanto aos critérios de formação, que pode ser aleatória ou por livre associação.

\section{Batalha}

As batalhas são encontros destinados à realização de duelos. Cada batalha tem um tema pré-definido disponibilizado previamente no ambiente virtual. A falta do jogador no dia da batalha corresponde à perda das patacas em disputa no respectivo dia.

\section{Auxiliares locais}


V Congresso Brasileiro de Informática na Educação (CBIE 2016)

Anais do XXVII Simpósio Brasileiro de Informática na Educação (SBIE 2016)

A cada batalha é designado dois auxiliares locais para a operacionalização das batalhas e o registro de todas as informações necessárias à execução do jogo em sala de aula. Os auxiliares são responsáveis por registrar os duelos (integrantes, ganhos e colaboração da plateia) e controlar o tempo.

\section{Os desafios}

Além da dinâmica das batalhas que induz o jogador a estudar antes das batalhas, foram criados questionários de fixação do conhecimento (questões de múltipla escolha) chamados desafios. Os desafios ficam disponíveis por quatro semanas no ambiente virtual imediatamente após a batalha correspondente. São dez questões aleatórias sobre os temas da batalha e o acerto de 6 ou mais questões enseja uma pataca.

\section{As missões}

As missões são atividades mais complexas feitas de forma individual e fora de sala de aula. Elas consistem em pesquisas, produção de perguntas para outros jogadores, respostas e avaliações das respostas dadas as perguntas feitas. Uma missão tem um período de uma semana, sendo dividida em atividades. Os alunos tem um tempo determinado para fazer cada atividade. Esta atividade tem um número diferenciado de patacas.

Para a execução das missões foi criado um segundo ambiente virtual para este fim denominado Milsa. No Milsa os jogadores criam perguntas abertas, que são respondidas pelos outros jogadores e depois avaliadas automaticamente por um sistema de correção automática. Por fim, o jogador que criou a pergunta avalia a resposta dada pelo outro jogador, a nota calculada automaticamente pelo Milsa, a justificativa do Milsa para a nota. Por fim o jogador deve justificar caso discorde da avaliação.

\section{Avaliação do engajamento e motivação dos alunos}

Para avaliar o engajamento e motivação dos alunos, foi empregado o questionário estruturado da Tabela 1.

Tabela 1: Itens do questionário

\begin{tabular}{|l|l|l|}
\hline ID & Questão & Tipo resposta \\
\hline Q1 & Qual a sua idade? & Valor \\
\hline Q2 & Que tipo(s) de computador(es) você tem acesso e utiliza em casa ou na Faculdade? & Múltipla escolha \\
\hline Q3 & Quantos jogos digitais você já jogou na sua vida? & Escolha única \\
\hline Q4 & Atualmente, quantas horas semanais você dedica a algum tipo de jogo digital? & Escolha única \\
\hline Q5 & Quais são as características dos jogos que mais lhe atrai? & Múltipla escolha \\
\hline Q6 & $\begin{array}{l}\text { Quanto tempo você gasta no estudo de FAC fora da sala de aula (na preparação de } \\
\text { uma batalha)? }\end{array}$ & Escolha única \\
\hline Q7 & Quais referências você tem consultado na preparação das batalhas? & Múltipla escolha \\
\hline Q8 & Os livros consultados são primordialmente: & Escolha única \\
\hline Q9 & $\begin{array}{l}\text { Você considera que os vídeos disponibilizados são úteis na sua preparação para as } \\
\text { batalhas? }\end{array}$ & Likert (5) \\
\hline Q10 & Você considera que a execução de exercícios após as batalhas (os desafios) são & Likert (5) \\
\hline
\end{tabular}


V Congresso Brasileiro de Informática na Educação (CBIE 2016)

Anais do XXVII Simpósio Brasileiro de Informática na Educação (SBIE 2016)

\begin{tabular}{|l|l|l|} 
& úteis na sua aprendizagem? & \\
\hline Q11 & Você vê FAC como um jogo? & Likert (5) \\
\hline Q12 & Como jogo, você se sente motivado a jogar as batalhas? & Likert (5) \\
\hline Q13 & Você sente que está aprendendo os conceitos de FAC através do jogo? & Likert (5) \\
\hline Q14 & Você considera FAC mais envolvente do que outras disciplinas já realizadas? & Likert (5) \\
\hline Q15 & Você considera FAC mais trabalhosa do que outras disciplinas já realizadas? & Likert (5) \\
\hline Q16 & $\begin{array}{l}\text { Você considera que FAC produz mais colaboração/cooperação do que outras } \\
\text { disciplinas já realizadas? }\end{array}$ & Likert (5) \\
\hline Q17 & Você recomendaria a um colega matricular-se na disciplina FAC? & Likert (5) \\
\hline
\end{tabular}

O questionário é dividido em duas partes: nos itens Q1 a Q8 são coletadas informações demográficas sobre o público alvo e nos itens Q9 a Q17 foram coletadas informações sobre a sensação do estudante quanto ao seu engajamento e motivação na disciplina gamificada. O questionário foi respondido por 27 dos 34 alunos da disciplina. Aplicado o critério de Alpha de Cronbach para as respostas aos itens não demográficos obteve-se o fator 0,70. Da análise dos itens demográficos, obteve-se:

- Idade média dos respondentes $=20,78$ anos.

- Aproximadamente $88 \%$ dos avaliados tem acesso a dois ou mais tipos de dispositivo, com $96,3 \%$ tem acesso a notebooks e $81,48 \%$ a smartphones.

- É um público que possui forte inserção nos jogos, onde 73,08\% já jogaram mais de 10 jogos, em torno de $50 \%$ ainda joga de 1 a 6 horas por semana e tem preferência por jogos com boas interfaces, com elementos de competição e também com elementos de colaboração.

- Em relação ao tempo gasto com FAC, 97,3\% dos respondentes estudam de 2 a 4 horas por batalha, como maior predominância $(77,78 \%)$ entre duas e três horas.

- Com relação às fontes de consulta, a predominância são os livros. Todos os três livros são consultados por mais de 50\% dos entrevistados (Tanenbaum 100\%, Stallings $66,7 \%$ e Patterson 51,9\%). O formato digital é o preferido (77,78\%).

Da análise dos itens referentes à sensação do estudante quanto ao seu engajamento e motivação na disciplina gamificada tem-se (considerando a escala de Likert de 1 a 5):

- Os estudantes avaliam FAC como jogo numa média de 3,63, com uma distribuição de $29,63 \%$ (3), $29,63 \%$ (4) e $25,93 \%$ (5).

- Os estudantes sentem-se motivados para as batalhas numa média de 3,04, com uma distribuição de 22,22\% (2), 22,22\% (3) e 40,74\% (4).

- A sensação de aprendizagem dos conceitos de FAC foi avaliada com uma média de 4,11, distribuídos em $18,52 \%$ (3), 51,5\% (4) e 29,63\% (5).

- A comparação da experiência dos estudantes com outras disciplinas do curso obteve uma média de 3,81 na avaliação do quanto a disciplina é mais envolvente, uma média de 3,85 na avaliação do quanto FAC é mais trabalhosa e uma média de 3,63 na avaliação do quanto FAC produz mais colaboração.

- Por fim, a recomendação da disciplina a um colega teve uma média de avaliação de 4,0 e uma distribuição de 25,93\% (3), 37,04\% (4) e 33,33\% (5). 
V Congresso Brasileiro de Informática na Educação (CBIE 2016)

Anais do XXVII Simpósio Brasileiro de Informática na Educação (SBIE 2016)

Da análise estatística também se identificou associação das respostas entre os itens: Q12 / Q14, Q14 / Q17, Q11 / Q12, Q12 /Q17 e Q11 / Q14.

A avaliação dos resultados indica que a disciplina ainda não é fortemente vista como um jogo (avaliação de 3,63). Apesar disso os jogadores estão fortemente envolvidos com a disciplina: estudam em torno de duas horas antes de cada batalha e consultam dois ou mais livros texto por batalha. Uma hipótese é: os estudantes são jogadores assíduos e com uma percepção mais refinada da parte de FAC que é jogo e da parte que é disciplina.

\section{Conclusões}

O uso da gamificação no ensino é aconselhável: (1) as novas gerações estão ambientadas com a dinâmica dos jogos, (2) os aspectos motivacionais da gamificação induzem os usuários a apenas jogarem, sem maiores comprometimentos e (3) a aprendizagem gamificada induz naturalmente o estudante a aprender com prazer.

Estas constatações figuraram tanto da observação in loco aos estudantes quanto dos dados analisados. Os estudantes dedicaram-se mais à disciplina: maior número horas de preparação para os conteúdos que serão vistos na sala de aula, maior uso da bibliografia disponível para o curso, empenho nos duelos e participação ativa nos duelos alheios. Também se observou uma melhoria acentuada da qualidade das questões elaboradas pelos estudantes para os duelos. Inicialmente de questões de conhecimento chegou-se a questões de aplicação (Bloom, 1956), a partir de 2/3 da disciplina.

A aferição do quanto o aluno teve uma maior aprendizagem do conteúdo da disciplina foi feita de forma indireta e qualitativa: o professor observou continuamente o teor das perguntas e respostas é identificou melhoras no nível de profundidade dos assuntos discutidos em relação aos últimos quatro semestres quando outra metodologia foi adotada (Freitas, Silva, \& Marsicano, 2016). Como a avaliação da aprendizagem ainda carece de maior sustentação, a continuação deste trabalho prevê o estudo e a utilização de métodos da área de Learning Analytics, mais adequados às metodologias ativas de aprendizagem.

Por fim, ainda como trabalho futuro, é necessário trabalhar a calibração dos elementos do jogo. Em dados momentos foi necessário "desmotivar" o jogador, pois ele estava gastando muitas horas em FAC em detrimento de outras disciplinas. Isto pode trazer efeitos negativos para o estudante. Uma proposta de solução para estes problemas é a utilização da teoria do Fluxo nas próximas versões do jogo.

\section{Referências}

Bell, S. (2010). Project-Based Learning for the 21st Century: Skills for the Future. The Clearing House, 83(2), 39-43.

Bloom, B. S. (Ed.). (1956). Taxonomy of Educational Objectives, the classification of educational goals - Handbook I: Cognitive Domain. New York: McKay.

Borges, S. d., Reis, H. M., Durelli, V. H., Bittencourt, I. I., Jaques, P. A., \& Isotani, S. (2013). Gamificação Aplicada à Educação: Um Mapeamento Sistemático. Anais do XXIV Simpósio Brasileiro de Informática na Educação (SBIE 2013), (pp. 254-263). 
V Congresso Brasileiro de Informática na Educação (CBIE 2016)

Anais do XXVII Simpósio Brasileiro de Informática na Educação (SBIE 2016)

Briggs, T. (2005). Techniques for active learning in CS courses. Journal of Computing Sciences in Colleges, 12(4), pp. 156-165.

Chou, Y.-k. (2015). Actionable Gamification: Beyond Points, Badges, and Leaderboards. Leanpub.

Ellwanger, C., Santos, C. P., \& Moreira, G. J. (2014). As Relações entre Gamificação, Padrões de Interface e Mobilidade no Desenvolvimento de Aplicações Educacionais. Anais do XXV Simpósio Brasileiro de Informática na Educação (SBIE 2014), (pp. 214-218).

Figueiredo, M., Paz, T., \& Junqueira, E. (2015). Gamificação e educação: um estado da arte das pesquisas realizadas no Brasil. Anais dos Workshops do IV Congresso Brasileiro de Informática na Educação (CBIE 2015), (pp. 1154 - 1163).

Fiorella, L., \& Mayer, R. E. (2014). Role of expectations and explanations in learning by teaching. Contemporary Educational Psychology, 39, 75-85.

Freitas, S. A., Silva, R. d., Lucena, T. F., RIBEIRO, E. D., LIMA, V. C., \& SILVA, R. M. (2016). Smart Quizzes in the Engineering Education. 49th Hawaii International Conference on System Sciences (HICSS), (p. 66). Koloa - HI - EUA.

Freitas, S. A., Silva, W. C., \& Marsicano, G. (2016). Using an Active Learning Environment to Increase Students' Engagement. 29th Conference on Software Engineering Education and Training (pp. 232-236). Dallas - TX: IEEE.

Kapp, K. M., Blair, L., \& Mesch, R. (2014). The Gamification of Learning and Instruction Fieldbook: San Francisco - CA: John Wiley \& Sons, Inc.

Kumar, J. M., \& Herger, M. (2013). Gamification at Work: Designing Engaging Business Software. Interaction Design Foundation.

McGonigal, J. (2011). Reality Is Broken: Why Games Make Us Better and How They Can Change the World. Penguin Books.

Patterson, D. A., \& Hennessy, J. L. (2013). Computer Organization and Design (5. ed.). Morgan Kaufmann.

Prensky, M. (2007). Digital Game-Based Learning. St. Paul - MN, USA: Paragon House.

Seixas, L. d., Gomes, A. S., Filho, I. J., \& Rodrigues, R. L. (2014). Gamificação como Estratégia no Engajamento de Estudantes do Ensino Fundamental. Anais do XXV Simpósio Brasileiro de Informática na Educação (SBIE 2014).

Stallings, W. (2012). Computer Organization and Architecture (9. ed.). Pearson.

Tanenbaum, A. S. (2013). Organização Estruturada de Computadores (6nd ed.). Pearson Education - Br.

Vianna, Y., Vianna, M., Medina, B., \& Tanaka, S. (2013). Gamification, Inc. - Como reinventar empresas a partir de jogos. Rio de Janeiro: MJV Press. 